\title{
Special issue of selected papers from the 15th Canadian Drosophila Research Conference, CANFLY XV 2019, held in Toronto, Ontario, Canada, on 9-13 June 2019
}

\author{
Guest Editors \\ Thomas J.S. Merritt, Laurentian University, Sudbury, ON, Canada \\ Andrew J. Simmonds, University of Alberta, Edmonton, AB, Canada \\ Rodrigo Fernandez-Gonzalez, University of Toronto, Toronto, ON, Canada
}

Amanda J. Moehring, Western University, London, ON, Canada 